\title{
What you want may not be what you like: A test of the aberrant salience hypothesis in schizophrenia risk
}

\author{
Lilian Yanqing Li ${ }^{1}$ (D) - Mayan K. Castro ${ }^{1}$ • Elizabeth A. Martin ${ }^{1}$
}

Published online: 7 July 2020

(C) The Psychonomic Society, Inc. 2020

\begin{abstract}
Motivational abnormalities represent a key area of dysfunction in individuals with, or at risk for, schizophrenia and severely limit broad domains of functioning in these populations. The aberrant salience hypothesis posits that motivational abnormalities are the result of an over-attribution of salience to nonpleasurable stimuli but an under-attribution of salience to pleasurable ones. Consequently, people "want" what they do not "like" but do not "want" what they "like." However, it is unclear how this hypothesis manifests in schizophrenia risk beyond monetary rewards. The current research provided a multimodal investigation of the aberrant salience hypothesis in people with elevated psychotic-like experiences (PLEs) who are at risk for developing psychosis. Study 1 examined the link between liking and incentive salience using a neurobiological indicator of incentive salience (contingent negative variation/CNV) in 23 PLEs and 21 Control participants. The PLEs group showed diminished $\mathrm{CNV}$ reactivity to pleasant (vs. neutral) social images, which was driven by an augmented response to neutral stimuli. Study 2 examined liking, incentive salience, and conscious wanting experience using a psychological indicator of incentive salience (positive spontaneous thoughts/PSTs) in 38 PLEs and 246 Control participants. The PLEs group showed diminished correspondence between liking, PSTs, and conscious wanting across diverse reward contexts. Collectively, individuals with PLEs overattribute salience to neutral stimuli and, to a lesser degree, under-attribute salience to rewards. Findings of the current research support abnormal salience attribution as a trait-like feature implicated in the pathophysiology and development of schizophrenia and provide valuable insights on research and treatment of this illness.
\end{abstract}

Keywords Aberrant salience $\cdot$ Psychotic-like experiences $\cdot$ Schizophrenia $\cdot$ Reward $\cdot$ Motivation

The pursuit of pleasure is a driving force of human life. From seeking out enjoyable sustenance and social bonds to endeavoring on a cherished career path, the motivation to pursue what we like is imperative to human survival, health, and well-being. Many individuals with schizophrenia, however, suffer from severe motivation dysfunction, which subsequently results in major impairments in functional outcomes and quality of life (Barch \& Dowd, 2010). Specifically, mounting research in this area paints a complex picture: people with schizophrenia inappropriately assign unremarkable,

Electronic supplementary material The online version of this article (https://doi.org/10.3758/s13415-020-00807-3) contains supplementary material, which is available to authorized users.

Elizabeth A. Martin

emartin8@uci.edu

1 Department of Psychological Science, 4201 Social and Behavioral Sciences Gateway, University of California, Irvine, CA 92697-7085, USA nonpleasurable objects and events with motivational properties, which is thought to underlie positive symptoms, such as hallucinations and delusions (Kapur, 2003; McCutcheon, AbiDargham, \& Howes, 2019). At the same time, they fail to appropriately generate motivation for pleasurable ones, which is thought to underlie negative symptoms, such as anhedonia and avolition (Barch \& Dowd, 2010; Strauss, Waltz, \& Gold, 2014). Critically, this pattern of motivational abnormalities extends to preclinical populations at risk for developing schizophrenia (Whitton \& Lewandowski, 2019), highlighting motivational abnormalities as enduring features of this illness. How can we reconcile this paradoxical motivational abnormality profile? The answer to this question could hold the key to understanding the pathophysiology and functional disability of schizophrenia.

The reward system is increasing recognized as a crucial player contributing to motivation abnormalities in schizophrenia. Three dissociable components comprise the reward system-liking, wanting, and learning - that guide motivated behaviors to obtain rewards (Berridge, 2007). "Liking" reflects 
the pleasure or hedonic impact of a reward, while "wanting" reflects the incentive salience attributed to the reward. Repeated encounters of a rewarding stimulus establish learned associations of cues that predict the reward (i.e., learning) and subsequently imbue these reward-predicting cues with incentive salience or wanting properties. Basic liking and wanting components are mediated by subcortical processes (e.g., striatum); these components could be further processed by prefrontal regions to be transferred into subjective feelings of conscious pleasure and desire (Berridge, 2007). Although disruption in any link in the chain of reward processing could give rise to motivational abnormalities, the incentive salience component has been proposed to play a particularly prominent role. The aberrant salience hypothesis was born out of a wealth of evidence showing abnormal dopamine transmission in schizophrenia (Heinz \& Schlagenhauf, 2010; Kapur, 2003) as well as accumulating findings demonstrating dopamine's direct effect on wanting, as opposed to liking or learning (Berridge, 2007). This hypothesis posits that schizophrenia is associated with elevated spontaneous dopamine release in the striatum, leading to aberrant attribution of salience to otherwise irrelevant stimuli. This hyperdopaminergic state may cause increased noise in the phasic dopamine release elicited by reward-predicting cues, leading to blunted adaptive salience attribution (Heinz \& Schlagenhauf, 2010; McCutcheon et al., 2019). Therefore, the aberrant salience hypothesis represents an integrative framework that bridges neurobiology (dopamine dysfunction) and phenomenology (motivational abnormality) of schizophrenia.

Extensive research has examined the aberrant salience hypothesis in patients with schizophrenia utilizing a variety of paradigms that span across multiple modes of analysis (e.g., self-report, behavioral, and neuroimaging). Overall, there is robust evidence supporting attenuated response to rewards and augmented response to neutral or nonrewarded stimuli (Heinz \& Schlagenhauf, 2010; Radua et al., 2015; Strauss et al., 2014). Nonetheless, prior research suffers from a number of limitations.

First, most studies did not separately examine neutral and reward conditions, but instead focused their analysis on the contrast between the two conditions (Radua et al., 2015). Therefore, it is unclear whether deficits in the reward vs. neutral contrast involves an over-attribution of salience to neutral stimuli or an under-attribution of salience to rewards or both. A refined examination of the aberrant salience hypothesis thus needs to account for the differential contribution of liking to abnormal salience attribution. Second, existing studies almost exclusively relied on monetary rewards, leaving the question open of whether salience for other types of rewards (e.g., social, physical, and cognitive) are equally deficient in schizophrenia. Although the reward system is generally thought to be modality independent (Berridge \& Kringelbach, 2015), there is evidence that individuals with schizophrenia are especially lacking in social motivation (Catalano, Heerey, \& Gold, 2018). Thus, examining salience attributed to a diverse range of reward types is necessary to delineate the full spectrum of motivation dysfunction. Last and most importantly, the use of patient samples might present critical confounds for the study of reward processing. Considering that most antipsychotic medications primarily target the dopamine transmission via blocking dopamine D2 receptors, these medications directly dampen incentive salience (Kapur, 2003). It is therefore unclear whether, or to what degree, incentive salience abnormalities observed in stably medicated schizophrenia patients result from "true" dysfunction or medication side effects. Several studies used medication-free, first-episode patients to circumvent medication confounds. However, this approach is also limited because these patients could be in a highly distressing psychotic state that likely involves acute alterations of dopamine transmission (Gold, Waltz, Prentice, Morris, \& Heerey, 2008; Laruelle \& Abi-Dargham, 1999). Because schizophrenia risk is expressed across a continuum of conditions ranging from individual differences to subclinical and clinical disorders (Kwapil \& Barrantes-Vidal, 2012, 2015; Nelson, Seal, Pantelis, \& Phillips, 2013), at-risk individuals prior to the appearance of clinical manifestations thus represent prime candidates for uncovering trait-like abnormalities associated with schizophrenia. Furthermore, at-risk individuals might be particularly valuable for testing the aberrant salience hypothesis, before the "delusional atmosphere" is transformed into rigid belief systems (Heinz \& Schlagenhauf, 2010).

Research on the aberrant salience hypothesis in at-risk populations has nonetheless been scant and inconclusive. Although there is emerging support for attenuated reactivity to reward (vs. neutral) stimuli among at-risk populations (Radua et al., 2015), conflicting findings showing a lack of group differences have also been reported (Kirschner et al., 2018; Yan et al., 2016). Moreover, prior research on at-risk individuals still has the same limitations of primarily focusing on reward versus neutral contrasts and monetary rewards, leaving unclear the precise mechanism of abnormal salience attribution across a wide range of reward types.

To address these gaps in the literature, the current research examined the aberrant salience hypothesis in schizophrenia risk over a series of two studies. Study 1 tested the link between liking and incentive salience for social rewards using a neurobiological indicator of incentive salience, the contingent negative variation (CNV). Study 2 extended Study 1 to a diverse array of social, physical, and cognitive rewards using a psychological indicator of incentive salience, positive spontaneous thoughts. Study 2 further tested the link between incentive salience and conscious wanting experiences. Both studies used participants with extremely elevated psychoticlike experiences (PLEs), which are less severe forms of psychotic symptoms shown to predict the development of 
psychosis (Chapman, Chapman, Kwapil, Eckblad, \& Zinser, 1994). In all, findings of the current research shed new lights on how the aberrant salience hypothesis manifests across various reward contexts in PLEs, which could be used to inform the development of early identification and intervention strategies.

\section{Study 1}

The main objective of Study 1 was to examine whether individuals with PLEs over-attribute incentive salience to neutral stimuli, but under-attribute incentive salience to socially rewarding stimuli. These key postulates of the aberrant salience hypothesis were examined in an electrophysiological paradigm apt to capture phasic dopamine transmission at the millisecond scale (Schultz, 2016). Specifically, we used a serial instrumental learning task. This task is commonly used to assess incentive salience and is typically referred to as the "S1-S2 design" in electrophysiological studies (Brunia, van Boxtel, \& Böcker, 2012). As detailed below, distinct cues (i.e., tones) were paired with later presentation of neutral vs. social-themed pleasant images, and reward outcomes (i.e., 3000 -ms vs. 300 -ms image viewing) depended on participants' performance on a speeded button press. Importantly, the auditory cue serves two functions: the onset of the tone functions as a warning stimulus (S1), while the offset of the tone functions as an imperative stimulus (S2) where a quick response is needed to obtain rewards. Despite originating from the same tone, these two functions manifest when the tone is sufficiently long. S1 carries the maximal prediction value because it reliably predicts S2 and reward. In contrast, S2 is a redundant predictor but carries the maximal incentive salience because it is a marker for immediate reward (Berridge, 2007). As such, the CNV, a fronto-central, negative deflection that peaks immediately before S2 is presented, is a good indicator of incentive salience attributed to the cue. Indeed, numerous studies have shown that $\mathrm{CNV}$ indexes motivated response preparation in the thalamo-cortico-striatal network (Brunia et al., 2012; Falkenstein, Hoormann, Hohnsbein, \& Kleinsorge, 2003; Fan et al., 2007). Notably, the CNV amplitude is larger in anticipation of rewards compared to nonrewards across a wide range of reward types (e.g., affective images and monetary rewards; Novak \& Foti, 2015; Simons, Öhman, \& Lang, 1979) and has been directly linked to activities in the mesolimbic reward circuit via combined EEG-fMRI investigation (Plichta et al., 2013).

We hypothesized that compared to the Control group, the PLEs group would show larger (i.e., more negative) CNV amplitudes, which would indicate an over-attribution of salience, in response to neutral images, but smaller (i.e., more positive) CNV amplitudes, which would indicate an underattribution of salience, in response to pleasant images.
Additionally, we hypothesized that CNV amplitudes would be significantly and negatively associated with self-reported liking of the images (i.e., valence and arousal ratings) and trait social pleasure experience in the Control group, which would imply the $\mathrm{CNV}$ as a valid indicator of incentive salience. These associations would be smaller (i.e., more positive) in the PLEs group, which would indicate an attenuated link between liking and incentive salience. To assess the specificity of the CNV, we also explored its association with trait nonsocial pleasure experience and current mood across groups.

\section{Method}

\section{Participants and measures}

Undergraduate students from a public, West Coast university were recruited using a psychometric high-risk approach. The group classification was based on self-reported responses, and there is ample evidence supporting the validity of this approach in identifying at-risk individuals (Lenzenweger, 2006). Specifically, individuals with extreme scores on these scales have an increased rate of future psychosis development (Chapman et al., 1994). In addition, scores on these scales have excellent correspondence with an interview-based psychosis risk assessment (i.e., the Structured Interview for Prodromal Syndromes; Miller et al., 2003), such that scores are moderately to strongly correlated with interview-rated symptoms (Cicero, Martin, Becker, Docherty, \& Kerns, 2014). Lastly, although the majority of the psychometric high-risk individuals do not eventually convert to schizophrenia, as is the case for all other high-risk approaches (e.g., clinical and familial high-risk; Fusar-Poli et al., 2013; Phillips \& Seidman, 2008), they nonetheless display dysfunctions in various domains and are thus worthy of studying in their own right (Kwapil \& Barrantes-Vidal, 2015; Lenzenweger, 2006).

In the current study, the PLEs group included 27 people who scored 1.5 SD above the mean on the short versions of the Perceptual Aberration Scale (PerAb) or Magical Ideation Scale (MagId; Winterstein, Silvia, et al., 2011b). The PerAb and MagId are designed to assess perceptual distortions and unusual beliefs, respectively, that parallel symptoms of hallucination and delusion (Chapman, Chapman, \& Raulin, 1978; Eckblad \& Chapman, 1983). The short versions of the scales were used due to their superior psychometric properties in ethnically diverse populations (Cicero, Martin, \& Krieg, 2019; Winterstein, Ackerman, Silvia, \& Kwapil, 2011a). The Control group included 25 people who scored less than $0.5 S D$ above the mean on the short versions of the PerAb, MagId, and Revised Social Anhedonia Scale. Power analysis indicated that a sample size of 25 participants per group was sufficient to test the primary hypothesis of the current study 
(see Supplementary Materials for more details). Further details on screening and recruitment are available in Martin, Li, and Castro (2020). ${ }^{1}$

Eight participants were excluded due to equipment failure ( $n=2$, one from each group) and low reliability of their CNV, defined as having fewer trials than needed to achieve a dependability point estimate of $0.7(n=6$, three from each group). The final sample consisted of 23 PLEs participants and 21 Control participants. Demographic information of the participants is shown in Table 1. Groups did not significantly differ in gender or ethnic composition, but the PLEs group was significantly younger than the Control group. Therefore, age was added as a covariate in all analyses. All results reported below remained largely unchanged when (a) without age as covariate and (b) excluding one Control participant who is responsible for the significant group difference in age (please refer to supplementary materials for details).

\section{Procedure and materials}

After providing informed consent, participants completed the following tasks in the order listed below in a quiet, temperature-controlled room. Upon completion, participants received course extra credit and monetary compensation. This study was approved by the relevant Institutional Review Board.

ERP paradigm Participants completed a serial instrumental learning task (Brunia et al., 2012) administered using Presentation Software (Neurobehavioral Systems, Inc., Berkeley, CA). There were two tone types: low frequency $(700 \mathrm{~Hz})$ and high frequency $(1200 \mathrm{~Hz})$. Each tone type was paired with an image category, either neutral or pleasant. Figure 1 illustrates the schematic of the trial structure. Each trial began with a centrally located fixation cross, with duration jittered between 1,400 ms and 2,000 ms. Next, a 2,000$\mathrm{ms}$ tone was presented, indicating whether a neutral or pleasant image would be presented subsequently. Participants were instructed to respond by pressing the space bar as quickly as possible at the offset of the tone. Depending on participants' performance, they then viewed an image for either 3,000 ms (i.e., responded within $250 \mathrm{~ms}$ ) or $300 \mathrm{~ms}$ (i.e., premature responses or response time greater than $250 \mathrm{~ms}$ ). Akin to winning money in monetary tasks, longer viewing time for pleasant images represents a rewarded outcome, whereas shorter viewing time for pleasant images as well as viewing neutral images represent a nonrewarded one.

\footnotetext{
${ }^{1}$ We also recruited 24 participants who scored $1.5 S D$ above the mean on the short version of the Revised Social Anhedonia Scale. They were not included in the current analysis due to low reliability of their CNV. Specifically, 190 trials were needed to achieve a dependability point estimate of 0.7 for participants with elevated social anhedonia, which exceeds the total number of trials.
}

Participants completed practice trials prior to the actual task to familiarize themselves with the timing and instructions. The actual task comprised a total of 80 trials: 40 neutral image trials and 40 pleasant image trials. Images were presented pseudo-randomly, such that a tone type was randomly selected, and then an image was randomly selected based on the chosen tone. The tone type-image category pairing was counterbalanced across participants. Images were selected from the International Affective Picture System (Lang, Bradley, \& Cuthbert, 2008). The neutral image was a photograph of a tissue box, which was presented 40 times. Pleasant images included images depicting affiliation and romance, with each image presented once. A complete list of images is shown in supplementary materials.

Image rating Participants provided valence $(1=$ extremely negative $; 9=$ extremely positive $)$ and arousal $(1=$ no bodily response; $9=$ strong bodily response) ratings using the SelfAssessment Manikin (Bradley \& Lang, 1994). ${ }^{2}$

Mood rating Current mood was assessed by four positively and four negatively valenced emotion adjectives of both high and low arousal (happy, excited, relaxed, calm, nervous, upset, sad, and fatigued). Ratings were made on a five-point scale ( $1=$ not at all; $5=$ extremely). Ratings within each valence category were averaged to yield a composite score for positive affect $(\mathrm{PA} ; \alpha=0.66)$ and negative affect (NA; $\alpha=0.67)$.

Pleasure experience Trait nonsocial pleasure experience was assessed using the Temporal Experience of Pleasure Scale (TEPS; Gard, Gard, Kring, \& John, 2006), and trait social pleasure experience was assessed using the Anticipatory and Consummatory Interpersonal Pleasure Scale (ACIPS; Gooding \& Pflum, 2014). The TEPS is composed of 10 items that measure anticipatory pleasure ("When ordering something off the menu, I imagine how good it will taste."; $\alpha=$ 0.71 ) and eight items that measure consummatory pleasure ("The smell of freshly cut grass is enjoyable to me."; $\alpha=$ 0.67). On the other hand, the 17-item ACIPS measures pleasure experience in the social domain ("I imagine how much fun it would be to go on vacation with a friend or someone I love."; $\alpha=0.87$ ). For both scales, participants rated how true each item described them generally on a six-point scale $(1=$ very false for me; $6=$ very true for me).

\section{EEG recording and processing}

Continuous EEG activity was recorded using the ANT-Neuro system (ANT-Neuro, Netherlands). Recording was from 32

\footnotetext{
${ }^{2}$ To minimize participant burden, they only rated a random subset of 30 images previously viewed from the serial instrumental learning task and from another, unrelated task reported in Martin et al. (2020).
} 
Table 1 Demographic characteristics and self-reported ratings of study 1 participants

\begin{tabular}{|c|c|c|c|}
\hline & $\begin{array}{l}\text { PLEs } \\
n=23\end{array}$ & $\begin{array}{l}\text { Control } \\
n=21\end{array}$ & Test statistics \\
\hline Female $n(\%)$ & $15(65.22)$ & $18(85.71)$ & $\chi^{2}(1, N=44)=1.49, p=0.22$ \\
\hline Age $M(S D)$ & $19.22(1.70)$ & $21.10(3.59)$ & $t(42)=2.25, p=0.03$ \\
\hline Race $n(\%)$ & & & $p=0.56$ (two-tailed Fisher's exact test) \\
\hline Asian & $13(56.52)$ & $8(38.10)$ & \\
\hline Caucasian & $4(17.39)$ & $5(23.81)$ & \\
\hline Latino/a & $4(17.39)$ & $7(33.33)$ & \\
\hline Other & $2(8.70)$ & $1(4.76)$ & \\
\hline \multicolumn{4}{|c|}{ Valence ratings $M(S D)$} \\
\hline Pleasant $^{\mathrm{a}}$ & $6.96(0.98)$ & $6.89(1.12)$ & $B(S E)=0.27(0.31), t(57.55)=0.87, p=0.39$ \\
\hline Neutral $^{b}$ & $5.00(0.60)$ & $5.27(1.27)$ & $B(S E)=-0.01(0.43), t(60.90)=-0.03, p=0.98$ \\
\hline \multicolumn{4}{|c|}{ Arousal ratings $M(S D)$} \\
\hline Pleasant $^{\mathrm{a}}$ & $4.85(1.54)$ & $4.28(1.34)$ & $B(S E)=0.62(0.48), t(61.00)=1.28, p=0.20$ \\
\hline Neutral $^{b}$ & $1.83(1.19)$ & $2.09(2.02)$ & $B(S E)=-0.17(0.67), t(61.00)=-0.26, p=0.80$ \\
\hline \multicolumn{4}{|c|}{ Current $\operatorname{mood} M(S D)$} \\
\hline Positive affect & $3.08(0.76)$ & $3.02(0.49)$ & $B(S E)=0.12(0.21), t(41)=0.56, p=0.58$ \\
\hline Negative affect & $2.36(0.69)$ & $2.06(0.61)$ & $B(S E)=0.20(0.20), t(41)=0.97, p=0.34$ \\
\hline \multicolumn{4}{|c|}{ Pleasure experience $M(S D)$} \\
\hline Anticipatory & $5.03(0.53)$ & $4.84(0.53)$ & $B(S E)=0.21(0.17), t(41)=1.26, p=0.22$ \\
\hline Consummatory & $5.01(0.64)$ & $4.76(0.61)$ & $B(S E)=0.27(0.20), t(41)=1.34, p=0.19$ \\
\hline Social & $5.18(0.53)$ & $5.24(0.42)$ & $B(S E)=-0.07(0.16), t(41)=-0.47, p=0.64$ \\
\hline
\end{tabular}

Note. PLEs $=$ psychotic-like experiences; $B=$ unstandardized coefficient.

${ }^{\mathrm{a}} n=20$ for the Control group

${ }^{\mathrm{b}} n=12$ for the PLEs group, $n=11$ for the Control group

$\mathrm{Ag} / \mathrm{AgCl}$ electrodes (FP1, FPz, FP2, Fz, F3, F4, F7, F8, FC5, FC1, FC2, FC6, Cz, C3, C4, T7, T8, CP1, CP2, CP5, CP6, Pz, P3, P4, P7, P8, POz, Oz, O1, O2, M1, M2) embedded in a cap and placed according to an expanded 10/20 system (American Electroencephalographic Society, 1994). Electrodes were referenced online to $\mathrm{CPz}$ and data was sampled at $1,000 \mathrm{~Hz}$.
All signal processing was conducted through EEGlab (Delorme \& Makeig, 2004). Trials for which participants responded prematurely were first removed, due to potential contamination of movement artifacts and the fact that $\mathrm{CNV}$ diminishes rapidly after a motor response is made (Brunia et al., 2012). Remaining data was down-sampled to $500 \mathrm{~Hz}$ and band-pass filtered with a second-order Butterworth filter

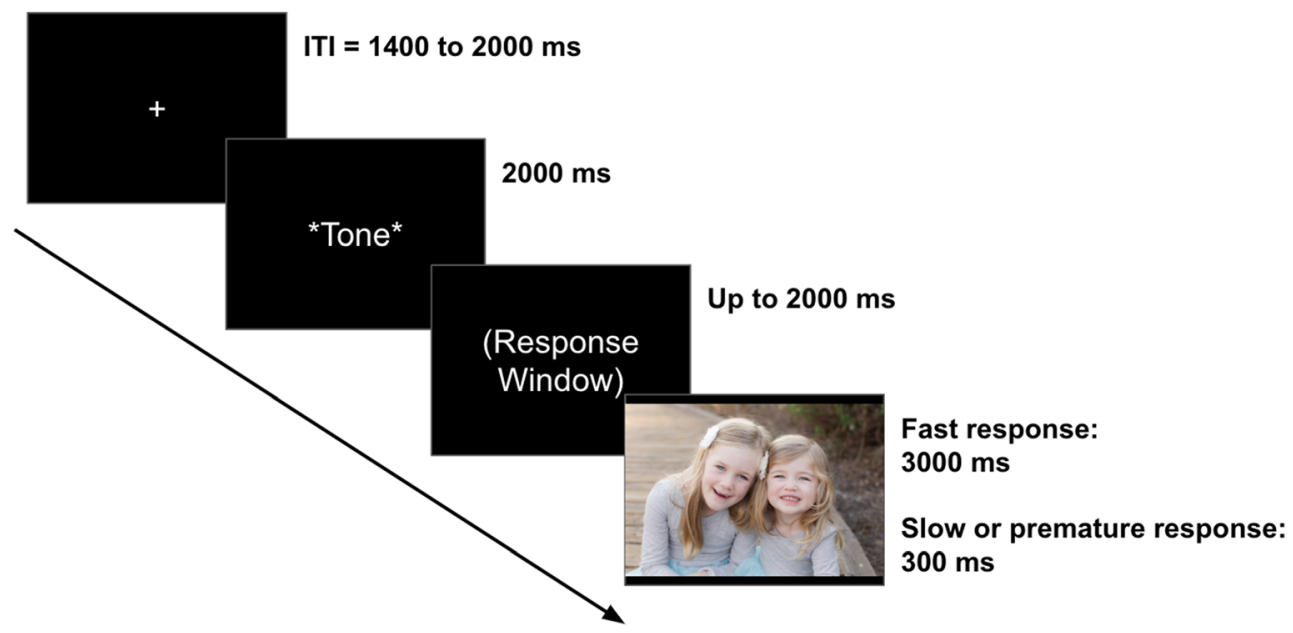

Fig. 1 Schematic representation of the trial structure. Note that the example image presented here is not part of the task stimuli 
from 0.1-30 Hz. Bad channels were identified as those containing extreme voltage fluctuations using Tukey's fences (i.e., with standard deviation more than 2 times the interquartile range from the third quartile) and replaced with whole head spline interpolation. Data was then re-referenced to average mastoids. Large muscle artifacts or extreme offsets were removed using a semi-automated procedure that identified voltages deflections of $\pm 200 \mu \mathrm{V}$. Next, independent component analysis (ICA) was applied to identify and remove eyeblink artifacts, defined as components containing extreme ICA activations and/or extreme fluctuations using Tukey's fences. After the ICA, 2200-ms epochs (including 200-ms prestimulus baseline) time-locked to the onset of the tones were extracted for each channel. Last, a moving window peak-to-peak artifact rejection procedure (window size $=200$ $\mathrm{ms}$; window step $=50 \mathrm{~ms}$ ) was used to remove epochs containing voltage deflections of $\pm 100 \mu \mathrm{V}$ in any of the channels used for CNV analysis.

Based on previous research (Brunia et al., 2012) and the topography of the CNV in the current study (averaged across all trials and participants), $\mathrm{CNV}$ amplitude was measured as the average voltage from $1,500 \mathrm{~ms}$ to $2,000 \mathrm{~ms}$ post-tone onset (or $500 \mathrm{~ms}$ pre-tone offset) at four fronto-central sites (Fz, FC1, FC2, and Cz). Reliability of the CNV was quantified by the generalization theory's index of dependability and calculated using the ERP Reliability Analysis (ERA) toolbox (Clayson \& Miller, 2017). Please refer to supplementary Table S1 for the psychometric properties of the CNV.

\section{Statistical analyses}

All analyses were performed using R (R Core Team, 2019). Separate linear regression models were used to examine group differences in current mood (PA and NA) and pleasure experience (anticipatory pleasure, consummatory pleasure, and social pleasure). For the ERP task, a series of preliminary analyses were first conducted. To examine valence and arousal ratings as well as viewing time of the images, separate Valence (Neutral vs. Pleasant) X Group (Control vs. PLEs) hierarchical linear models (HLMs) were conducted, with random intercepts of participants. The CNV was examined using a Valence (Neutral vs. Pleasant) X Group (Control vs. PLEs) HLM, with random intercepts of participants and of electrodes nested in participants. Last, the relation of self-reported ratings with CNV and potential moderation of group membership were examined using a Rating X Group (Control vs. PLEs) HLM, with random intercepts of participants and of electrodes nested in participants. HLMs were used to model participantspecific and electrode-specific variability through random effects. This provides us increased power to detect an effect as a

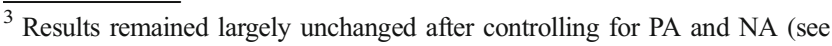
supplementary Table S2).
}

result of partitioning sources of variance from the error term. In addition, due to concerns over influential outliers, a sensitivity analysis was performed (a) using bootstrapping with 10,000 replications and (b) removing the influential outliers based on Cook's distance (Aguinis, Gottfredson, \& Joo, 2013). All models included age as a covariate.

\section{Results}

\section{Group characteristics}

Groups did not significantly differ in PA, NA, anticipatory pleasure, consummatory pleasure, or social pleasure (all $p \mathrm{~s}>$ 0.19; Table 1).

\section{Preliminary analyses}

As expected, pleasant images were rated as more positive (PLEs: $B(S E)=1.97(0.33), t(28.40)=6.00, p<0.001$; Control: $B(S E)=1.68(0.34), t(27.55)=4.88, p<0.001)$ and more arousing (PLEs: $B(S E)=3.00(0.54), t(61.00)=$ $5.50, p<0.001$; Control: $B(S E)=2.20(0.58), t(61.00)=3.83$, $p<0.001)$ than neutral images. Groups did not significantly differ in valence or arousal ratings for pleasant or neutral images (all $p \mathrm{~s}>0.32$; Table 1).

Also, viewing time did not significantly differ between groups or image valence categories (all $p \mathrm{~s}>0.14$ ). On average, images were viewed for $1822.88 \mathrm{~ms}(S D=643.38)$, which corresponds to a hit rate of $55.77 \%(S D=23.82)$. Thus, groups were matched in behavioral performance and were equally engaged in this task.

\section{CNV analyses}

Grand averaged waveforms are presented in Fig. 2. The Control group displayed larger $\mathrm{CNV}$ for pleasant than neutral images, $B(S E)=-2.12(0.47), t(306.00)=-4.54, p<0.001$. Relative to the Control group, the PLEs group displayed decreased CNV reactivity to pleasant (vs. neutral) images, $B(S E)$ $=2.76(0.65), t(306.00)=4.27, p<0.001$. Additionally, the PLEs group displayed trend-level larger CNV for neutral images than the Control group, $B(S E)=-3.10(1.64), t(44.35)=$ $-1.88, p=0.07$, but did not significantly differ from the Control group for pleasant images, $B(S E)=-0.34$ (1.64), $t(44.35)=-0.21, p=0.84 .^{3}$ Thus, the PLEs group showed attenuated reactivity to pleasant (vs. neutral) images, which was primarily driven by an increased response to neutral images.

As shown in Table 2, CNV amplitude was significantly predicted by valence and arousal ratings, as well as social pleasure experience, but not nonsocial pleasure experience, PA, or NA. For the Control group, greater arousal ratings ( $p$ $=0.02)$ and social pleasure $(p<0.001)$ predicted larger CNV 

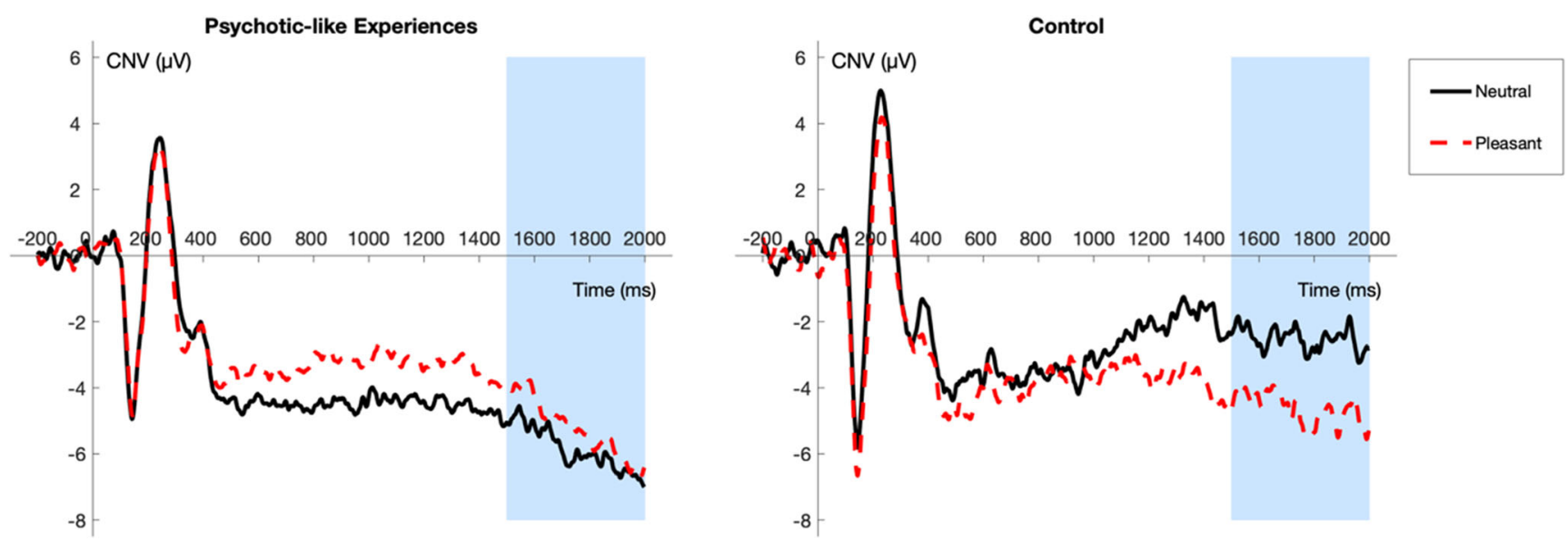

Fig. 2 Grand averaged CNV waveforms (average of Fz, FC1, FC2, and Cz) for the Psychotic-like Experiences group (left) and the Control group (right). Shaded area represents the time window used in the analysis

amplitude. This pattern was reversed for the PLEs group (all $p$ s < 0.02). Specifically, greater valence $(B(S E)=0.55(0.21)$, $t(233.77)=2.56, p=0.01)$ and arousal $(B(S E)=0.43(0.14)$, $t(240.15)=3.03, p=0.003)$ ratings predicted smaller $\mathrm{CNV}$ amplitude. At the same time, greater social pleasure failed to significantly predict CNV amplitude in the PLEs group, $B$ $(S E)=-0.13(1.80), t(39.00)=-0.07, p=0.94$. Taken togeth er, findings suggest that the PLEs group exhibited overattribution of salience to neutral stimuli.

\section{Sensitivity analysis}

Visual inspection of scatterplots identified some influential data points, we therefore performed a sensitivity analysis of CNV analyses using both bootstrapping and outlier deletion methods following established guidelines (Aguinis et al., 2013). For bootstrapping, bias-corrected estimates and 95\% confidence intervals were derived from 10,000 replications
(Hox, Moerbeek, \& van de Schoot, 2017). For outlier deletion, we followed a top-down approach in identifying influential outliers using Cook's distance by first examining potentially influential participants and then examining whether influential participants were driven by particular influential data points. Influential data points were first removed, and if the participant remained influential, the participant was then removed. Both methods produced highly similar results to our original analyses (see supplementary materials for full results). Thus, findings are not significantly impacted by the presence of outliers.

\section{Discussion}

To our knowledge, this is the first study to examine the differential contribution of liking to abnormal incentive salience attribution in PLEs. Consistent with prior work on schizophrenia and at-risk individuals (Radua et al., 2015), the PLEs

Table 2 Relation of self-reported ratings with Contingent Negative Variation (CNV)

\begin{tabular}{|c|c|c|c|c|c|c|}
\hline & \multicolumn{2}{|l|}{ Rating } & \multicolumn{2}{|c|}{ PLEs (vs. Control) } & \multicolumn{2}{|c|}{ Rating X PLEs (vs. Control) } \\
\hline & $B(S E)$ & $d f$ & $B(S E)$ & $d f$ & $B(S E)$ & $d f$ \\
\hline Valence ratings & $-0.21(0.25)$ & 244.07 & $-1.31(1.35)$ & 38.40 & $0.76(0.33)^{*}$ & 240.55 \\
\hline Arousal ratings & $-0.41(0.17)^{*}$ & 241.10 & $-1.50(1.38)$ & 38.98 & $0.84(0.22) * * *$ & 240.58 \\
\hline Positive affect & $-0.85(2.31)$ & 39.00 & $-1.56(1.62)$ & 39.00 & $-0.95(2.70)$ & 39.00 \\
\hline Negative affect & $0.85(1.94)$ & 39.00 & $-1.67(1.66)$ & 39.00 & $-2.05(2.48)$ & 39.00 \\
\hline Anticipatory pleasure & $-2.24(2.17)$ & 39.00 & $-1.51(1.66)$ & 39.00 & $2.62(2.98)$ & 39.00 \\
\hline Consummatory pleasure & $1.30(1.87)$ & 39.00 & $-1.69(1.66)$ & 39.00 & $-2.85(2.52)$ & 39.00 \\
\hline Social pleasure & $-8.63(2.36)^{* * *}$ & 39.00 & $-2.05(1.43)$ & 39.00 & $8.50(2.97)^{* *}$ & 39.00 \\
\hline
\end{tabular}

Note. Ratings were mean-centered. Age was included as a covariate (results not shown). PLEs $=$ psychotic-like experiences; $B=$ unstandardized coefficient; $S E=$ standard error; $d f=$ degrees of freedom.

$* * * p<0.001 ; * * p<0.01 ; * p<0.05$. 
group showed attenuated reactivity to pleasant relative to neutral images. We further showed that the attenuated reactivity was primarily driven by an exaggerated response to the neutral image. This over-attribution of salience to neutral stimuli in PLEs has been observed in the past literature using selfreported measures (Chun, Brugger, \& Kwapil, 2019; Cicero, Becker, Martin, Docherty, \& Kerns, 2013). There also is indirect behavioral evidence in PLEs pointing to increased novelty salience attributed to familiar yet task-irrelevant stimuli (e.g., latent inhibition tasks; Karcher, Martin, \& Kerns, 2015), as well as increased effort expenditure to obtain low-probability rewards (Ermel, Moran, Culbreth, \& Barch, 2019). Nevertheless, previous behavioral paradigms could not precisely address the role of incentive salience, given that 1) novelty salience is a property of the sensory feature rather than motivational value (Schultz, 2016); and 2) effort-based decision-making heavily relies on higher-order resources to integrate action and outcome (Kurniawan, Guitart-Masip, \& Dolan, 2011).

It is important to note that while the current task relies on successful learning of the prediction value of the tone before it can trigger incentive salience properties, the current findings are unlikely the result of a learning deficit. This is because the $\mathrm{CNV}$ was measured immediately before the imperative stimulus S2, a signal that carries maximal incentive salience rather than prediction value (Berridge, 2007). Furthermore, the CNV response was significantly predicted by self-reported liking in both Control and PLEs groups: whereas greater liking was associated with larger CNV amplitude in the Control group, it was associated with smaller CNV amplitude in the PLEs group. This is inconsistent with a learning deficit, since a failure to learn the prediction value in the PLEs group would result in a lack of association between self-reported liking and CNV amplitude. Relatively preserved learning ability has also been observed in patients with schizophrenia, especially where learning occurs gradually, over a number trials (Gold et al., 2008; Strauss et al., 2014). Thus, converging lines of evidence support the conclusion that motivation dysfunction is the chief product of abnormal salience attribution.

However, contrary to our prediction, the PLEs group failed to show reduced response to pleasant images. It is possible that under-attribution of salience to rewards might be particularly relevant to negative symptoms (Barch \& Dowd, 2010; Strauss et al., 2014). Alternatively, pleasant images might not be a particularly attractive reward to notably produce incentive salience. This explanation is supported by the comparable viewing time, and hence hit rates, for pleasant and neutral images, indicating that the rewarded outcome (i.e., longer viewing time of pleasant images) did not generate a large enough behavioral reinforcement of faster reaction times leading to increased hit rates for pleasant vs. neutral images. This limitation is addressed in Study 2, which tested the aberrant salience hypothesis using activities that span across a variety of contexts, some of which are commonly enjoyed and performed on a regular basis.

\section{Study 2}

The main objectives of Study 2 were to extend Study 1 findings across diverse reward contexts, and to further examine whether abnormal incentive salience attribution translates into impaired conscious wanting experiences in individuals with PLEs. Of note, the activation of incentive salience may not be necessarily accompanied by conscious wanting experiences. For the subjective experience of desire to occur, additional prefrontal and related cortical processing is needed to transform incentive salience that is largely subcortical (Berridge, 2007; Kringelbach, 2005). Prefrontal abnormalities are a hallmark of schizophrenia as well as its risk (Fioravanti, Bianchi, \& Cinti, 2012; Gee et al., 2012; Hooker et al., 2014; Nelson et al., 2013), so it remains unclear whether motivational dysfunction can be directly linked to abnormal salience attribution, as opposed to abnormalities in higher-order cortical processing.

In Study 2, we used positive spontaneous thoughts, that is, pleasant thoughts that arise without explicit intent, as a psychological indicator of incentive salience. There is evidence suggesting that people ascribe meaning and significance (i.e., salience) to the content of spontaneous, rather than deliberate, thoughts (Morewedge, Giblin, \& Norton, 2014). Furthermore, positive spontaneous thoughts have been shown to causally mediate the link between liking and conscious wanting: greater liking of a activity engendered greater positivity of spontaneous thoughts which in turn prompted greater motivation to engage in that activity in the future (Rice \& Fredrickson, 2017).

Participants completed an activities task used in Rice and Fredrickson (2017). Specifically, participants were asked to indicate how positive their spontaneous thoughts had been in the past 24 hours toward ten common daily activities. Participants also rated their levels of positive emotions while engaging in each activity in the past week (excluding the previous day) as an index of liking as well as how much they wanted to do each activity in the next 24 hours as an index of conscious wanting. We hypothesized that the Control group would show positive links between positive emotions, thought positivity, and conscious wanting, which would imply positive spontaneous thoughts as a valid indicator of incentive salience. On the basis of the aberrant salience hypothesis (i.e., over- vs. under-attribution of salience to neutral versus reward stimuli), we also hypothesized that the PLEs group would not differ significantly from the Control group in the overall levels of positive emotions, thought positivity, and conscious wanting but would show attenuated associations between them. 
Table 3 Demographic characteristics of Study 2 participants

\begin{tabular}{llll}
\hline & $\begin{array}{l}\text { PLEs } \\
n=38\end{array}$ & $\begin{array}{l}\text { Control } \\
n=246\end{array}$ & Test statistics \\
\hline $\begin{array}{lll}\text { Female } n(\%)^{\mathrm{a}} \\
\text { Age } M(S D)\end{array}$ & $\begin{array}{l}28(73.68) \\
\text { Race } n(\%)\end{array}$ & $\begin{array}{l}194(78.86) \\
20.82(4.32)\end{array}$ & $\begin{array}{l}\chi^{2}(1, N=283)=0.31, p=0.58 \\
t(282)=0.47, p=0.64\end{array}$ \\
$\begin{array}{l}\text { Asian } \\
\text { African American }\end{array}$ & $13(34.21)$ & $85(34.55)$ & $p=0.18$ (two-tailed Fisher's exact test) \\
Caucasian & $2(5.26)$ & $5(2.03)$ & \\
Latino/a & $5(13.16)$ & $62(25.20)$ & \\
Other & $13(34.21)$ & $78(31.71)$ & $16(6.50)$ \\
\hline
\end{tabular}

Note. PLEs = psychotic-like experiences.

${ }^{a}$ One Control participant identified as neither male nor female.

\section{Method}

\section{Participants}

Participants were 38 PLEs and 246 Control individuals, a sample size that was sufficiently powered to test the primary hypothesis (see supplementary materials for more details). Screening criteria was identical as Study 1. Groups did not differ in gender, age, or ethnic composition (Table 3).

\section{Procedure and materials}

After providing informed consent, participants completed the activities task administered through Qualtrics (Qualtrics, Provo, UT) and received course credit as compensation. Following the procedure developed by Rice and Fredrickson (2017), participants were provided a list of 10 common daily activities (e.g., exercising or being physically active, learning something new, and socializing). They first rated the frequency of spontaneous thoughts within the past 24 hours for each activity using a 0-100 scale. For activities having at least one spontaneous thought, they reported the levels of positivity and negativity of the thoughts $(0=$ not at all; $10=$ extremely $)$. Next, participants rated whether they engaged in each activity during the week before the previous day, and if they indicated yes to the question, their levels of positive and negative emotions experienced during the activity $(0=$ not at all; $10=$ extremely). Participants then reported how much they wanted to do each activity in the next 24 hours regardless of practical issues $(0=$ not at all; $10=$ extremely $)$. Last, participants rated their levels of autonomy over each activity $(0=$ no autonomy; $10=$ total autonomy $)$, last time they did each activity $(0=$ not at all/never/several years ago; $10=$ today), next time they planned on doing each activity $(0=$ never/in several years; $10=$ today $)$, and general frequency of doing each activity $(0=$ never $; 10=$ everyday). Participants also completed some questionnaires unrelated to the current study's hypotheses and thus are not reported here. The relevant Institutional Review Board approved this study.

\section{Statistical analyses}

All analyses were performed using R (R Core Team, 2019). Group differences in positive emotions, thought positivity, and conscious wanting were first examined using HLMs with random intercepts of participants. To examine the relation of liking with incentive salience and the potential moderation of group membership, a HLM was conducted with withinsubject positive emotions (level 1), between-subjects positive emotions (level 2), and Group (Control vs. PLEs; level 2) along with its interaction with positive emotions (both within and between components) as predictors, and thought positivity as outcome. We specified random intercepts of participants and random slopes of within-subject positive emotions. To examine the relation of incentive salience with conscious wanting and the potential moderation of group membership, a HLM was conducted with within-subject thought positivity (level 1), between-subjects thought positivity (level 2), and Group (Control vs. PLEs; level 2) along with its interaction with thought positivity (both within and between components) as predictors, and conscious wanting as outcome, controlling for levels of autonomy and general activity frequency. We specified random intercepts of participants and random slopes of within-subject thought positivity. Last, using a similar model, an exploratory analysis was conducted examining the relation of thought positivity with time to next activity and the potential moderation of group membership, controlling for time since last activity. For all models, level 2 continuous variables were grand-mean centered, and level 1 continuous variables were person-mean centered. 
Table 4 Parameter estimates of Study 2 analyses

\begin{tabular}{|c|c|c|c|c|c|c|}
\hline \multirow[t]{2}{*}{ Fixed effects } & \multirow[t]{2}{*}{$B(S E)$} & \multirow[t]{2}{*}{$t$} & \multirow[t]{2}{*}{$d f$} & \multirow[t]{2}{*}{$p$} & \multicolumn{2}{|l|}{$95 \% \mathrm{CI}$} \\
\hline & & & & & Lower & Upper \\
\hline \multicolumn{7}{|l|}{ ST positivity $\sim$ Positive emotions } \\
\hline Intercept & $6.07(0.06)$ & 107.04 & 272.01 & $<0.001$ & 5.96 & 6.18 \\
\hline PLEs (vs. Control) & $-0.02(0.16)$ & -0.10 & 285.40 & 0.92 & -0.32 & 0.29 \\
\hline Positive emotions (within) & $0.76(0.02)$ & 34.49 & 234.06 & $<0.001$ & 0.72 & 0.80 \\
\hline Positive emotions (within) X PLEs (vs. Control) & $-0.12(0.06)$ & -2.05 & 237.10 & 0.04 & -0.24 & -0.005 \\
\hline Positive emotions (between) & $0.76(0.05)$ & 15.16 & 280.51 & $<0.001$ & 0.66 & 0.86 \\
\hline Positive emotions (between) X PLEs (vs. Control) & $-0.004(0.12)$ & -0.03 & 297.90 & 0.97 & -0.23 & 0.23 \\
\hline \multicolumn{7}{|l|}{ Conscious wanting $\sim$ ST positivity } \\
\hline Intercept & $2.93(0.28)$ & 10.33 & 2522.11 & $<0.001$ & 2.37 & 3.48 \\
\hline PLEs (vs. Control) & $0.11(0.22)$ & 0.49 & 282.07 & 0.62 & -0.32 & 0.53 \\
\hline ST positivity (within) & $0.80(0.02)$ & 32.55 & 546.23 & $<0.001$ & 0.75 & 0.85 \\
\hline ST positivity (within) X PLEs (vs. Control) & $-0.21(0.06)$ & -3.34 & 419.92 & $<0.001$ & -0.33 & -0.08 \\
\hline ST positivity (between) & $0.45(0.07)$ & 6.74 & 306.60 & $<0.001$ & 0.32 & 0.58 \\
\hline ST positivity (between) X PLEs (vs. Control) & $0.01(0.16)$ & 0.07 & 313.19 & 0.95 & -0.30 & 0.32 \\
\hline Autonomy & $0.19(0.02)$ & 9.45 & 2545.27 & $<0.001$ & 0.15 & 0.23 \\
\hline General Frequency & $0.19(0.03)$ & 6.06 & 2637.80 & $<0.001$ & 0.13 & 0.25 \\
\hline \multicolumn{7}{|l|}{ Time to next activity $\sim$ ST positivity } \\
\hline Intercept & $3.62(0.13)$ & 27.60 & 2521.23 & $<0.001$ & 3.36 & 3.87 \\
\hline PLEs (vs. Control) & $0.05(0.10)$ & 0.49 & 281.70 & 0.63 & -0.15 & 0.25 \\
\hline ST positivity (within) & $0.06(0.01)$ & 5.28 & 284.32 & $<0.001$ & 0.04 & 0.09 \\
\hline ST positivity (within) X PLEs (vs. Control) & $0.06(0.03)$ & 2.00 & 244.60 & 0.046 & 0.001 & 0.13 \\
\hline ST positivity (between) & $0.04(0.03)$ & 1.21 & 281.34 & 0.23 & -0.02 & 0.10 \\
\hline ST positivity (between) X PLEs (vs. Control) & $-0.09(0.08)$ & -1.12 & 291.43 & 0.26 & -0.24 & 0.06 \\
\hline Time since last activity & $0.58(0.01)$ & 39.35 & 2621.90 & $<0.001$ & 0.55 & 0.61 \\
\hline
\end{tabular}

Note. PLEs = psychotic-like experiences; $\mathrm{ST}=$ spontaneous thoughts; $B=$ unstandardized coefficient; $S E=$ standard error; $d f=$ degrees of freedom; $\mathrm{CI}=$ confidence interval.

\section{Results}

As expected, the PLEs group did not differ from the Control group in their average levels of positive emotions $(B(S E)=$ $-0.22(0.20), t(284.36)=-1.06, p=0.29)$, thought positivity $(B(S E)=-0.33(0.20), t(281.92)=-1.62, p=0.11)$, or conscious wanting $(B(S E)=-0.10(0.24), t(282.00)=-0.42, p=$ $0.68)$. Further inquiry into the links between positive emotions, thought positivity, and conscious wanting revealed significant group differences (Table 4; Fig. 3). For the Control group, greater within-subject and between-subjects positive emotions were associated with greater thought positivity; greater within-subject and between-subjects thought positivity were associated with greater conscious wanting (all $p \mathrm{~s}<$ 0.001). Therefore, the expected pattern of associations was found for variations across activities within each participant as well as individual differences across participants in the Control group. Compared with the Control group, the PLEs group showed reduced association between within-subject positive emotions and thought positivity $(p=0.04)$, as well as between within-subject thought positivity and conscious wanting $(p<0.001){ }^{4}$ Therefore, the PLEs group showed attenuated relations between liking, incentive salience, and conscious wanting, such that greater liking toward an activity was associated with diminished increase in incentive salience for the activity, which in turn was associated with diminished increase in conscious desire to do that activity.

An intriguing finding emerged from the exploratory analysis relating thought positivity to time to next activity (Table 4; Fig. 3). As expected, greater within-subject thought positivity was associated with shorter time planned to next engage in a given activity in the Control group $(p<0.001)$. Interestingly, this association was larger in the PLEs group relative to the Control group ( $p=0.046$ ), such that greater thought positivity towards an activity was associated with an

\footnotetext{
${ }^{4}$ Results remained the same after controlling for trait PA and trait NA (see supplementary Table S11). Trait affect was measured using the Positive and Negative Affect Schedule (Watson, Clark, \& Tellegen, 1988) within 2 weeks before completing the activities task ( $\alpha$ s for both PA and NA $=0.87$ ).
} 

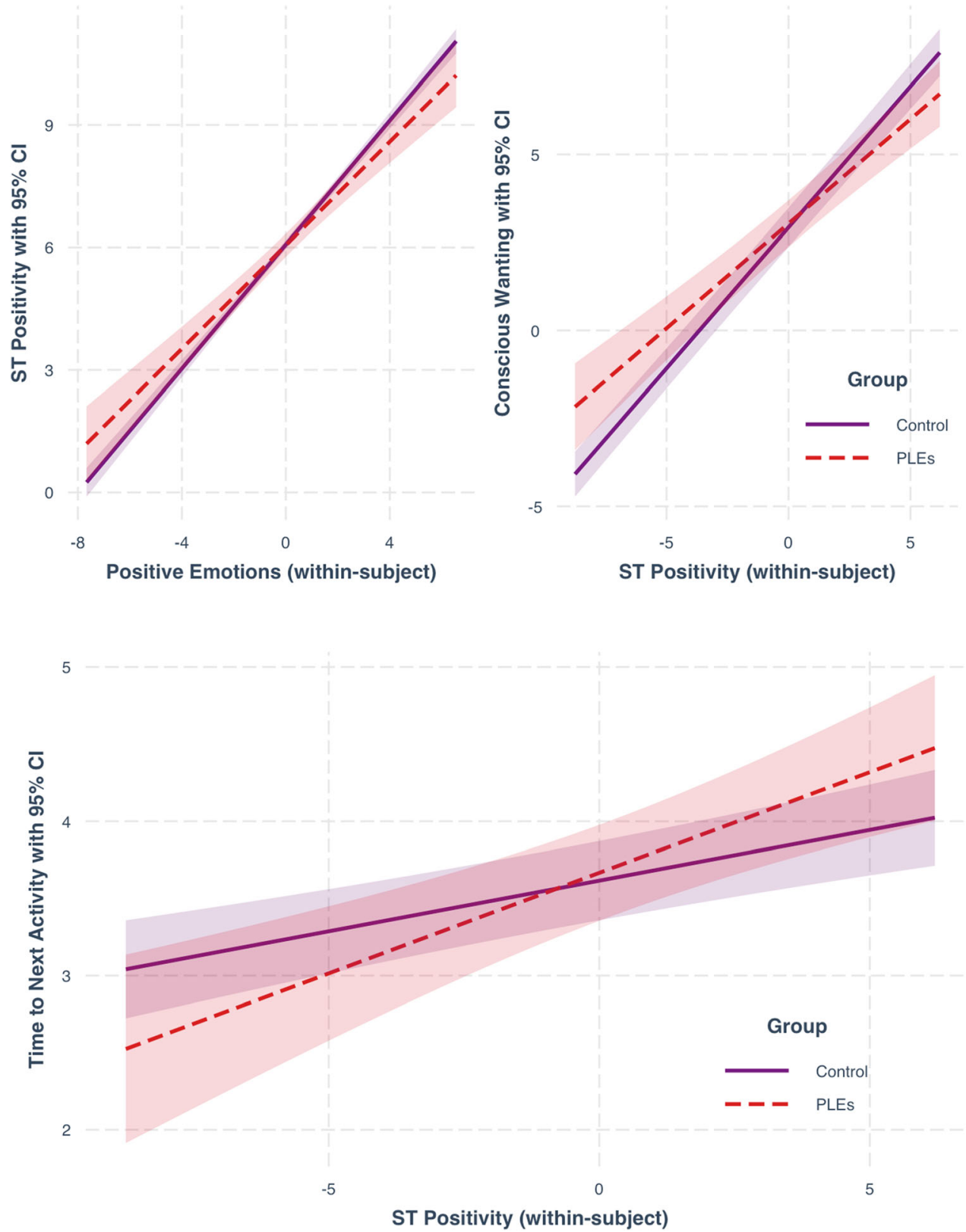

Fig. 3 The Psychotic-like Experiences (PLEs) group displayed attenuated association between positive emotions, spontaneous thoughts (ST) positivity, and conscious wanting (top), but displayed augmented

exaggerated increase in the urgency to do that activity. Thus, the PLEs group appears to weigh incentive salience more heavily into future activity planning.

\section{Discussion}

Study 2 extended Study 1 by testing the aberrant salience hypothesis across diverse social, physical, and cognitive rewards experienced in everyday life. We showed that relative to the Control group, the PLEs group displayed an attenuated relation between liking and incentive salience, rather than a difference in the average levels, supporting the differential association between ST positivity and time to next activity (bottom) compared to the Control group

contribution of liking to abnormal incentive salience attribution. Furthermore, we showed that the incentive salience abnormalities alone can result in dysfunctional conscious wanting for the activities. Because conscious wanting experience requires additional prefrontal and related cortical processes to transform striatum-mediated incentive salience (Berridge, 2007), coupled with a large body of literature showing executive functioning deficits in schizophrenia (Fioravanti et al., 2012), many previous studies focused on higherorder abnormalities to explain motivation dysfunction. For instance, the value representation hypothesis argues that motivational abnormalities are consequent to deficits 
in maintaining and updating values underpinned by orbital and dorsal prefrontal structures leading to a failure to appropriately use value to direct behavior (Barch \& Dowd, 2010; Gold et al., 2008; Strauss et al., 2014). In addition, the effort computation hypothesis maintains that the evaluation of whether a certain reward value is worth the effort to obtain that reward is compromised, a process that is underpinned by the anterior cingulate cortex (Barch \& Dowd, 2010; Strauss et al., 2014). Although dysfunctions in value representation and effort computation may very well play a role in motivational abnormalities, these hypotheses are very general in scope, often referring to broad areas of deficits that have less to do with specific components of reward processing. We showed, for the first time, that abnormal attribution of incentive salience was related to motivational disturbances.

At first glance, the finding that PLEs showed greater influence of incentive salience on future activity planning seems to be contradictory to the attenuated relations between incentive salience and conscious wanting. However, whereas conscious wanting taps into near-term reward motivation (i.e., in the next 24 hours), planning for future rewards involves long-term decision-making that spans from today to several years later. Research has shown that while evaluating immediate rewards preferentially engages the limbic structures (e.g., striatum), evaluating distant rewards is a function of both limbic and prefrontal inputs (McClure, Laibson, Loewenstein, \& Cohen, 2004). Preference for long-term over short-term rewards is contingent on prefrontal control overriding the limbic drive for immediacy, and a failure to activate prefrontal resources would thereby result in impulsive behaviors (McClure et al., 2004). Therefore, our results suggest that the PLEs may lack the prefrontal control needed for deliberate planning for future rewards, contributing to an over-weighing of the striatal input and subsequently a preference for sooner, more immediate rewards. This finding is consistent with the literature showing patients with schizophrenia discount future rewards more steeply than healthy controls (Gold et al., 2008) as well as display elevated risk-taking behaviors such as substance use, aggression, and suicidality (Ouzir, 2013). Although offering promising insights into the role of incentive salience in maladaptive behaviors, this study relies on subjective reports and is correlational in nature. Whether incentive salience predicts actual behavioral outcomes will be an important question for the future.

\section{General discussion}

The abnormal salience attribution postulated by the aberrant salience hypothesis elegantly reconciles the seemingly paradoxical coexistence of hyper-motivation and hypo-motivation in schizophrenia. Nevertheless, explanatory gaps remain due to methodological limitations of previous research. In two studies, the current research provided a rigorous test of the aberrant salience hypothesis in individuals with elevated PLEs that examined neurobiological (Study 1) and psychological (Study 2) indicators of incentive salience across a diverse array of social, physical, and cognitive rewards. Findings provided converging support for PLEs associated with over-attribution of salience to neutral stimuli and, to a lesser degree, under-attribution of salience to rewarding ones, thereby highlighting incentive salience abnormalities as a trait-like feature implicated in the pathophysiology and development of schizophrenia.

The current findings have important implications for research and treatment. The past 20 years have witnessed an explosion of research on the reward system in schizophrenia - a cursory PsycINFO search using "reward" and "schizophrenia" as keywords yielded 1,131 articles between 2000 and 2019, which is almost six times the amount produced in the prior 70 years combined (200 articles between 1930 and 1999). While this bourgeoning interest in the reward system brings forth exciting avenues for understanding schizophrenia, many prior studies present limitations that make it difficult to pinpoint the most relevant mechanism of dysfunction. As detailed above, a number of theories and empirical investigations primarily focus on domain-general processes (e.g., executive functioning) that, although important, do not directly address reward-specific components. For studies that did examine the reward system, many failed to consider the multicomponent nature of the reward system, typically targeting one aspect of this system (e.g., liking or wanting) without investigating the interactions between them (e.g., how liking differentially contributes to wanting abnormalities). One major contribution of the current research is that incentive salience abnormalities are contingent on the levels of liking, with divergent effects (i.e., over- vs. under-attribution) observed for neutral vs. pleasant stimuli. This effect extends to salience-related neurobiological and psychological markers and across various reward contexts, further reinforcing the value of examining the interaction between reward components in schizophrenia and its associated risk.

As abnormal salience attribution lies at the heart of motivational abnormalities and maladaptive behaviors, the most effective therapeutic approach should directly strengthen the correspondence between liking and incentive salience. This points to the insufficiency of a solely pharmacological treatment approach: while antipsychotic medications directly dampen incentive salience, they do so irrespective of the levels of liking and hence may serve to dampen adaptive motivations for rewarding stimuli (Kapur, 2003). Additional psychosocial treatments, such as cognitive behavioral therapy (CBT), are needed to discriminate non-rewarding triggers from rewarding ones and challenge inaccurate experiences and beliefs. Furthermore, implicit and explicit reinforcements may be needed to boost incentive salience for rewards. For example, life and social skills training should emphasize applying the learned skills in real world situations. The pleasures associated with new achievements (e.g., successfully carrying out a 
conversation) may engender increased incentive salience to the adaptive behaviors and associated cues (e.g., conversation partner and facial expressions). In addition, some form of token economy could be applied as an explicit reinforcement of adaptive behaviors, such as encouragement from family members and clinicians. Testing these approaches in at-risk individuals to prolong or prevent the onset of psychosis will be of great significance, given that current single-component preventative approaches (e.g., antipsychotic medications, omega-3 supplements, and CBT) are not particularly efficacious (Davies et al., 2018). Conversely, integrated psychological interventions containing CBT, social skills training, cognitive remediation, and psychoeducation have shown to be promising (Davies et al., 2018), so it would be fruitful to incorporate the aforementioned practices into existing intervention programs.

Despite the current research offering intriguing substantive and clinical insights, several important questions remain. First, we specifically focused on individuals with elevated PLEs. The lack of significant negative symptoms in our participants might have contributed to a less robust finding for attenuated salience attribution to rewards, but we believe that the content of the stimuli might have played a bigger role. Relatedly, besides schizophrenia risk, we did not assess other aspects of psychopathology, such as depression and anxiety. Notably, preliminary evidence suggests that the abnormal salience attribution observed in the current research might be specific to schizophrenia risk, with the current findings remaining largely unchanged after controlling for state and trait affect. Nevertheless, how aberrant salience may operate in those with pronounced negative features, as well as whether depression and anxiety play a role in abnormal salience attribution, remain to be tested in future studies. Second, it is important to note that the aberrant salience hypothesis extends beyond reward processing (Howes \& Nour, 2016; WintonBrown, Fusar-Poli, Ungless, \& Howes, 2014), and the current research cannot directly address processing in response to salient, but nonrewarding (e.g., aversive), stimuli. However, considering that at least some aspects of the incentive and aversive salience are underpinned by a common motivational salience mechanism (Berridge \& Kringelbach, 2016), it is tempting to speculate that abnormal salience attribution might also underlie dysfunctional aversive processing in schizophrenia (Balog, Somlai, \& Kéri, 2013; Jensen et al., 2008). Few studies to date have examined this topic and further studies are clearly warranted. Third, the generalizability of our findings is limited by our use of college samples. However, college students offer distinct advantages in testing the aberrant salience hypothesis, because they are at a critical transitional period that not only marks the peak age of onset for schizophrenia but also presents numerous academic and life stressors that may precipitate PLEs. Furthermore, having a college education does not preclude liability for psychosis, with clinically meaningful PLEs having been observed in college populations (Cicero et al., 2014). Nevertheless, future studies may wish to examine abnormal salience attribution using samples drawn from the community and clinical settings. Last, the current findings should be cautiously interpreted within the confines of relatively small sample sizes. While further replication studies with larger sample sizes are needed, the robustness of our main findings are confirmed by the sensitivity analysis as well as the estimation of true power (Replicability Index $=81.56 \%$ ).

In conclusion, the current research provided a novel, multimodal investigation of the aberrant salience hypothesis in individuals with PLEs, who were found to display patterns of abnormal salience attribution that are consistent with this hypothesis. Findings emphasize the differential contribution of liking to incentive salience abnormalities, which may be an important target for future research and treatment.

Open practices statement The data that support the findings of the current research are available at https://osf.io/7sdx3. Neither of the studies reported in the current article was formally preregistered.

\section{References}

Aguinis, H., Gottfredson, R. K., \& Joo, H. (2013). Best-Practice Recommendations for Defining, Identifying, and Handling Outliers. Organizational Research Methods, 16, 270-301.

American Electroencephalographic Society. (1994). Guideline thirteen: guidelines for standard electrode position nomenclature. Journal of Clinical Neurophysiology, 11, 111-3.

Balog, Z., Somlai, Z., \& Kéri, S. (2013). Aversive conditioning, schizotypy, and affective temperament in the framework of the salience hypothesis. Personality and Individual Differences, 54, 109112.

Barch, D. M., \& Dowd, E. C. (2010). Goal representations and motivational drive in schizophrenia: The role of prefrontal-striatal interactions. Schizophrenia Bulletin, 36, 919-934.

Berridge, K. C. (2007). The debate over dopamine's role in reward: The case for incentive salience. Psychopharmacology, 191, 391-431.

Berridge, K. C., \& Kringelbach, M. L. (2015). Pleasure Systems in the Brain. Neuron, 86, 646-664.

Berridge, K. C., \& Kringelbach, M. L. (2016). From pleasure to happiness. "Liking" and "wanting" in mind and brain. In L. F. Barrett, M. Lewis, \& J. M. Haviland-jones (Eds.), The Handbook of Emotion, 4th edition (pp. 133-145). New York, NY, US: Guilford Press.

Bradley, M. M., \& Lang, P. J. (1994). Measuring emotion: The selfassessment manikin and the semantic differential. Journal of Behavior Therapy and Experimental Psychiatry, 25, 49-59.

Brunia, C. H. M., van Boxtel, G. J. M., \& Böcker, K. B. E. (2012). Negative slow waves as indices of anticipation: The Bereitschaftspotential, the Contingent Negative Variation, and the Stimulus-Preceding Negativity. In S. J. Luck \& E. S. Kappenman (Eds.), The Oxford Handbook of Event-Related Potential Components (pp. 189-207). New York, NY, US: Oxford University Press. 
Catalano, L. T., Heerey, E. A., \& Gold, J. M. (2018). The Valuation of Social Rewards in Schizophrenia. Journal of Abnormal Psychology, 127, 602-611.

Chapman, L. J., Chapman, J. P., Kwapil, T. R., Eckblad, M., \& Zinser, M. C. (1994). Putatively Psychosis-Prone Subjects 10 Years Later. Journal of Abnormal Psychology, 103, 171-183.

Chapman, L. J., Chapman, J. P., \& Raulin, M. L. (1978). Body-image aberration in schizophrenia. Journal of Abnormal Psychology, 87, 399-407.

Chun, C. A., Brugger, P., \& Kwapil, T. R. (2019). Aberrant Salience Across Levels of Processing in Positive and Negative Schizotypy. Frontiers in Psychology, 10, 2073.

Cicero, D. C., Becker, T. M., Martin, E. A., Docherty, A. R., \& Kerns, J. G. (2013). The role of aberrant salience and self-concept clarity in psychotic-like experiences. Personality Disorders, 4, 33-42.

Cicero, D. C., Martin, E. A., Becker, T. M., Docherty, A. R., \& Kerns, J. G. (2014). Correspondence between psychometric and clinical high risk for psychosis in an undergraduate population. Psychological Assessment, 26, 901-915.

Cicero, D. C., Martin, E. A., \& Krieg, A. (2019). Differential Item Functioning of the Full and Brief Wisconsin Schizotypy Scales in Asian, White, Hispanic, and Multiethnic Samples and Between Sexes. Assessment, 26, 1001-1013.

Clayson, P. E., \& Miller, G. A. (2017). ERP Reliability Analysis (ERA) Toolbox: An open-source toolbox for analyzing the reliability of event-related brain potentials. International Journal of Psychophysiology, 111, 68-79.

Davies, C., Cipriani, A., Ioannidis, J. P. A., Radua, J., Stahl, D., Provenzani, U., ... Fusar-Poli, P. (2018). Lack of evidence to favor specific preventive interventions in psychosis: a network meta-analysis. World Psychiatry, 17, 196-209.

Delorme, A., \& Makeig, S. (2004). EEGLAB: an open source toolbox for analysis of single-trial EEG dynamics including independent component analysis. Journal of Neuroscience Methods, 134, 9-21.

Eckblad, M., \& Chapman, L. J. (1983). Magical ideation as an indicator of schizotypy. Journal of Consulting and Clinical Psychology, 51, 215-225.

Ermel, J. A., Moran, E. K., Culbreth, A. J., \& Barch, D. M. (2019). Psychotic like experiences as part of a continuum of psychosis: Associations with effort-based decision-making and reward responsivity. Schizophrenia Research, 206, 307-312.

Falkenstein, M., Hoormann, J., Hohnsbein, J., \& Kleinsorge, T. (2003). Short-term mobilization of processing resources is revealed in the event-related potential. Psychophysiology, 40, 914-923.

Fan, J., Kolster, R., Ghajar, J., Suh, M., Knight, R. T., Sarkar, R., \& McCandliss, B. D. (2007). Response anticipation and response conflict: An event-related potential and functional magnetic resonance imaging study. Journal of Neuroscience, 27, 2272-2282.

Fioravanti, M., Bianchi, V., \& Cinti, M. E. (2012). Cognitive deficits in schizophrenia: An updated metanalysis of the scientific evidence. BMC Psychiatry, 12, 64

Fusar-Poli, P., Borgwardt, S., Bechdolf, A., Addington, J., RiecherRössler, A., Schultze-Lutter, F., ... Yung, A. (2013). The psychosis high-risk state: A comprehensive state-of-the-art review. Archives of General Psychiatry, 70, 107-120.

Gard, D. E., Gard, M. G., Kring, A. M., \& John, O. P. (2006). Anticipatory and consummatory components of the experience of pleasure: A scale development study. Journal of Research in Personality, 40, 1086-1102.

Gee, D. G., Karlsgodt, K. H., van Erp, T. G. M., Bearden, C. E., Lieberman, M. D., Belger, A., ... Cannon, T. D. (2012). Altered age-related trajectories of amygdala-prefrontal circuitry in adolescents at clinical high risk for psychosis: A preliminary study. Schizophrenia Research, 134, 1-9.
Gold, J. M., Waltz, J. A., Prentice, K. J., Morris, S. E., \& Heerey, E. A. (2008). Reward processing in schizophrenia: A deficit in the representation of value. Schizophrenia Bulletin, 34, 835-847.

Gooding, D. C., \& Pflum, M. J. (2014). The assessment of interpersonal pleasure: Introduction of the Anticipatory and Consummatory Interpersonal Pleasure Scale (ACIPS) and preliminary findings. Psychiatry Research, 215, 237-243.

Heinz, A., \& Schlagenhauf, F. (2010). Dopaminergic Dysfunction in Schizophrenia: Salience Attribution Revisited. Schizophrenia Bulletin, 36, 472-485.

Hooker, C. I., Benson, T. L., Gyurak, A., Yin, H., Tully, L. M., \& Lincoln, S. H. (2014). Neural activity to positive expressions predicts daily experience of Schizophrenia-Spectrum symptoms in adults with high social anhedonia. Journal of Abnormal Psychology, 123, 190-204

Howes, O. D., \& Nour, M. M. (2016). Dopamine and the aberrant salience hypothesis of schizophrenia. World Psychiatry, 15, 3-4.

Hox, J. J., Moerbeek, M., \& van de Schoot, R. (2017). Multilevel Analysis: Techniques and Analysis (Third edit). New York, NY, US: Routledge. doi:https://doi.org/10.4324/9781315650982

Jensen, J., Willeit, M., Zipursky, R. B., Savina, I., Smith, A. J., Menon, M., ... Kapur, S. (2008). The formation of abnormal associations in schizophrenia: Neural and behavioral evidence. Neuropsychopharmacology, 33, 473-479.

Kapur, S. (2003). Psychosis as a state of aberrant salience: A framework linking biology, phenomenology, and pharmacology in schizophrenia. American Journal of Psychiatry, 160, 13-23.

Karcher, N. R., Martin, E. A., \& Kerns, J. G. (2015). Examining associations between psychosis risk, social anhedonia, and performance of striatum-related behavioral tasks. Journal of Abnormal Psychology, 124, 507-518.

Kirschner, M., Hager, O. M., Muff, L., Bischof, M., Hartmann-Riemer, M. N., Kluge, A., ... Kaiser, S. (2018). Ventral Striatal Dysfunction and Symptom Expression in Individuals with Schizotypal Personality Traits and Early Psychosis. Schizophrenia Bulletin, 44, 147-157.

Kringelbach, M. L. (2005). The human orbitofrontal cortex: Linking reward to hedonic experience. Nature Reviews Neuroscience, 6, 691702.

Kurniawan, I. T., Guitart-Masip, M., \& Dolan, R. J. (2011). Dopamine and effort-based decision making. Frontiers in Neuroscience, 5, 81.

Kwapil, T. R., \& Barrantes-Vidal, N. (2012). Schizotypal Personality Disorder: An Integrative Review. In T. A. Widiger (Ed.), The Oxford Handbook of Personality Disorders (pp. 437-477). Oxford University Press.

Kwapil, T. R., \& Barrantes-Vidal, N. (2015). Schizotypy: Looking back and moving forward. Schizophrenia Bulletin, 41, S366-S373.

Lang, P. J., Bradley, M. M., \& Cuthbert, B. N. (2008). International affective picture system (IAPS): Affective ratings of pictures and instruction manual. Technical Report A-8. University of Florida, Gainesville, FL.

Laruelle, M., \& Abi-Dargham, A. (1999). Dopamine as the wind of the psychotic fire: New evidence from brain imaging studies. Journal of Psychopharmacology, 13, 358-371.

Lenzenweger, M. F. (2006). Schizotypy an organizing framework for schizophrenia research. Current Directions in Psychological Science, 15, 162-166.

Martin, E. A., Li, L. Y., \& Castro, M. K. (2020). Electrophysiological responses to images ranging in motivational significance: Abnormal EPN and LPP amplitudes associated with schizophrenia-spectrum disorder risk. Scientific Reports, 10, 4578.

McClure, S. M., Laibson, D. I., Loewenstein, G., \& Cohen, J. D. (2004). Separate neural systems value immediate and delayed monetary rewards. Science, 306, 503-507. 
McCutcheon, R. A., Abi-Dargham, A., \& Howes, O. D. (2019). Schizophrenia, Dopamine and the Striatum: From Biology to Symptoms. Trends in Neurosciences, 42, 205-220.

Miller, T. J., McGlashan, T. H., Rosen, J. L., Cadenhead, K., Ventura, J., McFarlane, W., ... Woods, S. W. (2003). Prodromal Assessment with the Structured Interview for Prodromal Syndromes and the Scale of Prodromal Symptoms: Predictive Validity, Interrater Reliability, and Training to Reliability. Schizophrenia Bulletin, 29, 703-715.

Morewedge, C. K., Giblin, C. E., \& Norton, M. I. (2014). The (perceived) meaning of spontaneous thoughts. Journal of Experimental Psychology: General, 143, 1742-1754.

Nelson, M. T., Seal, M. L., Pantelis, C., \& Phillips, L. J. (2013). Evidence of a dimensional relationship between schizotypy and schizophrenia: A systematic review. Neuroscience and Biobehavioral Reviews, 37, 317-327.

Novak, K. D., \& Foti, D. (2015). Teasing apart the anticipatory and consummatory processing of monetary incentives: An eventrelated potential study of reward dynamics. Psychophysiology, 52, $1470-1482$

Ouzir, M. (2013). Impulsivity in schizophrenia: A comprehensive update. Aggression and Violent Behavior, 18, 247-254.

Phillips, L. K., \& Seidman, L. J. (2008). Emotion processing in persons at risk for schizophrenia. Schizophrenia Bulletin, 34, 888-903.

Plichta, M. M., Wolf, I., Hohmann, S., Baumeister, S., Boecker, R., Schwarz, A. J., ... Brandeis, D. (2013). Simultaneous EEG and fMRI reveals a causally connected subcortical-cortical network during reward anticipation. Journal of Neuroscience, 33, 14526-14533.

R Core Team. (2019). R: A language and environment for statistical computing. Vienna, Austria: R Foundation for Statistical Computing.

Radua, J., Schmidt, A., Borgwardt, S., Heinz, A., Schlagenhauf, F., McGuire, P., \& Fusar-Poli, P. (2015). Ventral striatal activation during reward processing in psychosis: A neurofunctional meta-analysis. JAMA Psychiatry, 72, 1243-1251.

Rice, E. L., \& Fredrickson, B. L. (2017). Do positive spontaneous thoughts function as incentive salience? Emotion, 17, 840-855.
Schultz, W. (2016). Dopamine reward prediction-error signalling: A twocomponent response. Nature Reviews Neuroscience, 17, 183-195.

Simons, R. F., Öhman, A., \& Lang, P. J. (1979). Anticipation and Response Set: Cortical, Cardiac, and Electrodermal Correlates. Psychophysiology, 16, 222-233.

Strauss, G. P., Waltz, J. A., \& Gold, J. M. (2014). A review of reward processing and motivational impairment in schizophrenia. Schizophrenia Bulletin, 40, S107-S116.

Watson, D., Clark, L. A., \& Tellegen, A. (1988). Development and validation of brief measures of positive and negative affect: the PANAS scales. Journal of Personality and Social Psychology, 54, 1063 1070

Whitton, A. E., \& Lewandowski, K. E. (2019). Reward processing and social functioning in psychosis. In K. E. Lewandowski \& A. A. Moustafa (Eds.), Social Cognition in Psychosis (pp. 177-200). Elsevier.

Winterstein, B. P., Ackerman, T. A., Silvia, P. J., \& Kwapil, T. R. (2011a). Psychometric properties of the wisconsin schizotypy scales in an undergraduate sample: Classical test theory, item response theory, and differential item functioning. Journal of Psychopathology and Behavioral Assessment, 33, 480-490.

Winterstein, B. P., Silvia, P. J., Kwapil, T. R., Kaufman, J. C., ReiterPalmon, R., \& Wigert, B. (2011b). Brief assessment of schizotypy: Developing short forms of the Wisconsin Schizotypy Scales. Personality and Individual Differences, 51, 920-924.

Winton-Brown, T. T., Fusar-Poli, P., Ungless, M. A., \& Howes, O. D. (2014). Dopaminergic basis of salience dysregulation in psychosis. Trends in Neurosciences, 37, 85-94.

Yan, C., Wang, Y., Su, L., Xu, T., Yin, D., Zhi, Fan, M. Xia, ... Chan, R. C. K. (2016). Differential mesolimbic and prefrontal alterations during reward anticipation and consummation in positive and negative schizotypy. Psychiatry Research - Neuroimaging, 254, 127-136.

Publisher's note Springer Nature remains neutral with regard to jurisdictional claims in published maps and institutional affiliations. 